MIDPI

MOL2NET, International Conference Series on Multidisciplinary Sciences

sciforum,

\title{
Engineering Ferrous-ferric oxide nanocomposites for heavy metal ions removal from aqueous solution
}

\author{
Sahebeh Tamaddoni Moghaddam ${ }^{a}$ \\ ${ }^{a}$ Department of Materials Science \& Engineering, College of Engineering \& Applied Science, \\ University of Wisconsin-Milwaukee, 3200 N. Cramer Street, Milwaukee, WI, 53211, USA
}

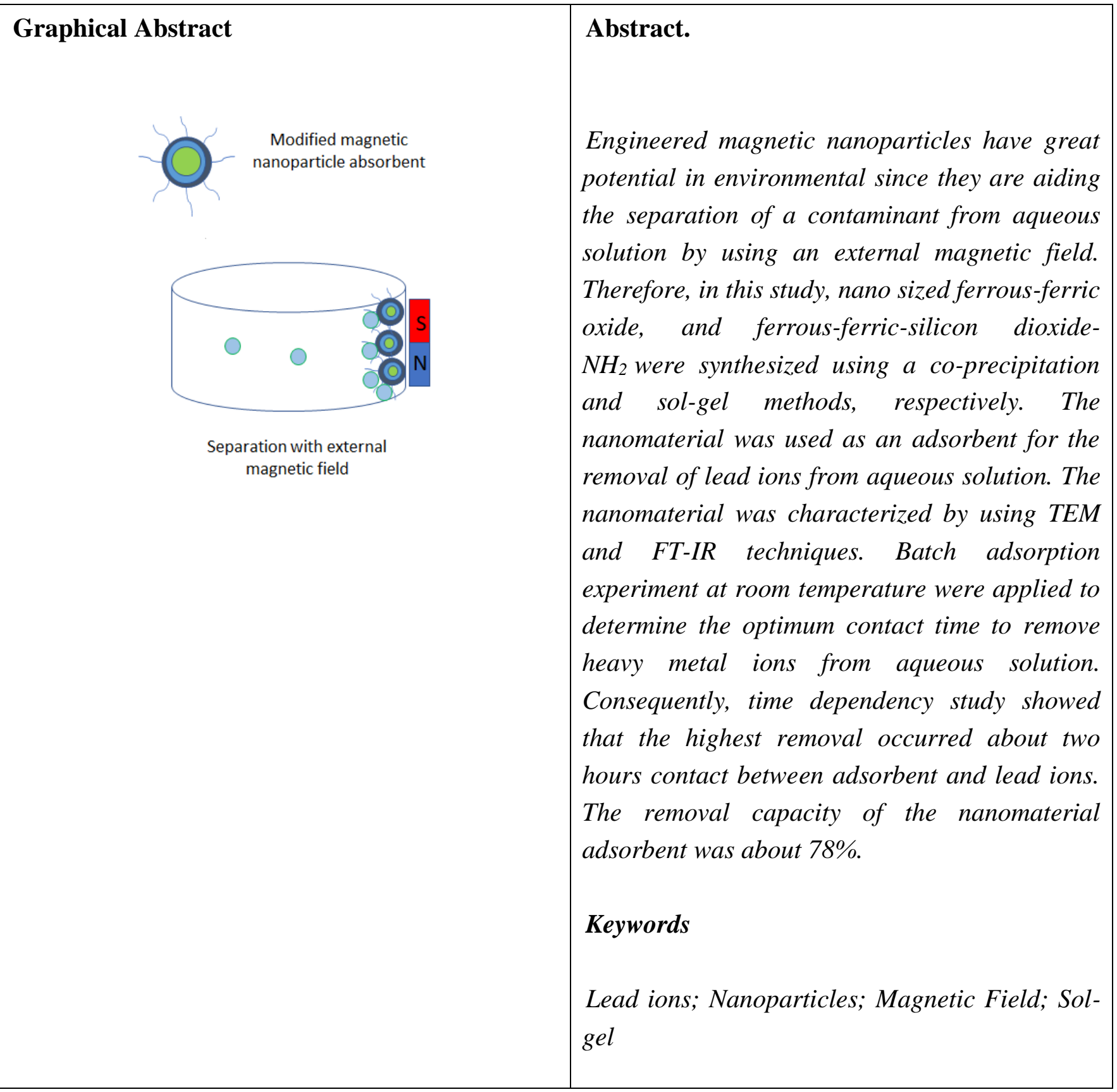




\section{Introduction}

The environment and all the life on earth are challenging with a very serious threat because of high levels of pollution that increase in the world. Some of these pollutions can be degraded biologically, but metal ions do not easily change into harmless end products. Existence of heavy metal ions contaminants in water are a major risk to disadvantaged communities, since they attitude a substantial health risk to people that cannot protected safe drinking water [1-3]. Among heavy metal ions, lead ion is one of the highly toxic and carcinogenic material that has incited the scientific community to generate new methods in the removal of substance from aqueous solution. Several methods such as chemical precipitation, ionexchange process, electrolytic method, adsorption, and reverse osmosis processes stated before to eliminate heavy metal ions from water [4].

Nanomaterials have attracted considerable research interest in environmental engineering due to their nanoparticle size, high surface areas, and enhanced activity sites on the surface. Ferrous-ferric oxide nanoparticles have demonstrated to be a good candidate to remove of heavy metal ions from aqueous solutions. Since they have large surface area and high reactivity specificity [4].

To protect the surface of ferrous-ferric oxide nanoparticles against oxidation, a surface modifier such as silica can be used [5,6]. Furthermore, surface modification of ferrous-ferric oxide is used to expand the performance of nanoparticles to reduce the aggregation of ferrous-ferric oxide nanoparticles [4]. Therefore, in this research, nano sized ferrous-ferric oxide, and ferrous-ferric-silicon dioxide- $\mathrm{NH}_{2}$ nanocomposites were synthesized by using a co-precipitation and sol-gel methods, respectively for the removal of Lead ions from aqueous solution. The nanomaterial was characterized by using TEM and FT-IR techniques. Atomic absorption spectroscopy analysis was applied to analyze the concentration of the lead ions from aqueous solutions. Time dependency study showed that the highest removal capacity has occurred about two hours contact time between adsorbent and lead ions. The removal capacity of the nanomaterial adsorbent was $78 \mathrm{wt} \%$.

\section{Materials and Methods}

Surface morphology of the nanoparticles were investigated by TEM Jeol JEM-2100. FT-IR spectra were recorded on a Nicolet iS5 spectrophotometer. The adsorption capacity of the absorbent was measured with the Atomic absorption spectrometer-Shimadzu. Lead standard solution of 1,000 ppm was purchased from Fisher Scientific and was used to prepare the initial solution. Iron (II) chloride tetrahydrate, iron (III) chloride hexahydrate, ammonia solution, tetraethyl orthosilicate, ethanol, 3aminopropyltriethoxysilane, were purchased from Sigma-Aldrich company.

The ferrous-ferric dioxide nanoparticles synthesized by co-precipitation method by the reaction of ferric and ferrous in ammonia solution. Briefly, iron (III) chloride (0.017 mol) and iron (II) chloride $(0.011 \mathrm{~mol})$ dispersed in deionized water and sonicated. Then ammonia solution added quickly into the mixture under nitrogen atmosphere and stirred for 2 hours with mechanical stirrer. Finally, nanoparticles washed several times with distilled water and dried in oven $[5,6]$.

For the synthesis of ferrous-ferric-silicon dioxide- $\mathrm{NH}_{2}$ nanoparticles, in first step, $0.5 \mathrm{~g}$ of ferrousferric dioxide nanoparticle dispersed in ethanol solution and sonicated, then stirred followed by addition of ammonia solution. Then tetraethyl orthosilicate solution added and stirred overnight. Modified nanoparticles collected and washed with water and dried in oven for the next step. In second step, $0.5 \mathrm{~g}$ 
of ferrous-ferric-silicon dioxide dispersed in ethanol and sonicated. Then 3-aminopropyltriethoxysilane added in the solution and the mixture stirred at room temperature under nitrogen atmosphere. The ferrous-ferric-silicon dioxide- $\mathrm{NH}_{2}$ nanoparticle separated by external magnetic field and washed with distilled water several times and dried in vacuum [4].

\section{Results and Discussion}

FT-IR analysis

Surface morphology of ferrous-ferric-silicon dioxide- $\mathrm{NH}_{2}$ NPs were characterized by FTIR spectroscopy (Figure 1). As shown in the figure, the band at $592 \mathrm{~cm}-1$ is attributed to $\mathrm{Fe}-\mathrm{O}$ bending vibration mode [7]. The strong absorption band at $1072 \mathrm{~cm}-1$ is relate to the asymmetrical stretching vibration of $\mathrm{Si}-\mathrm{O}-\mathrm{Si}[5,6]$.The presence of $\mathrm{C}-\mathrm{H}$ groups of 3-aminopropyltriethoxysilane were established by stretching vibrations at 2800 2900 cm-1 in the FTIR spectrum [8,9]. These results indicated that the surface of ferrous-ferric oxide nanoparticles was modified with silicon dioxide- $\mathrm{NH}_{2}$ groups as well.

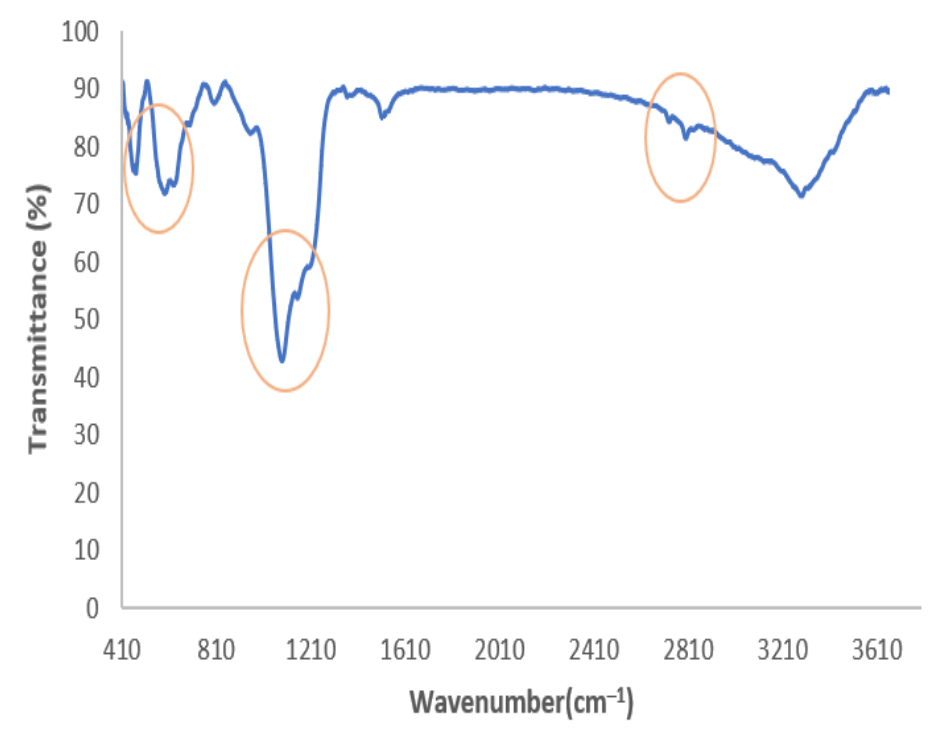

Figure 1. FT-IR spectra of ferrous-ferric-silicon dioxide- $\mathrm{NH}_{2}$ nanoparticles

TEM analysis

TEM image of synthesized ferrous-ferric-silicon dioxide- $\mathrm{NH}_{2}$ NPs are shown in figure 2. By modification of the surface of ferrous-ferric oxide nanoparticles, you see less aggregation in TEM image, and the average size of nanoparticles is below $100 \mathrm{~nm}$. In addition, TEM image shows a core-shell structure of ferrous-ferric-silicon dioxide- $\mathrm{NH}_{2}$ nanoparticles. 


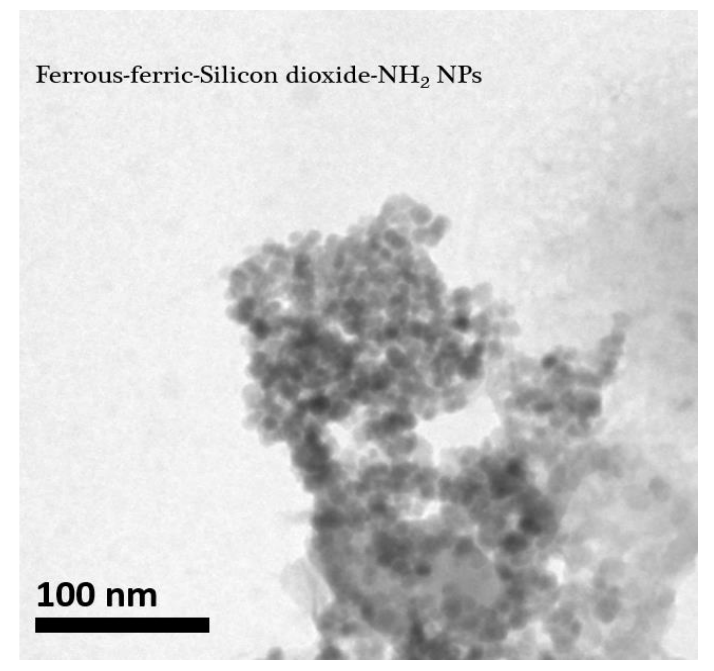

Figure 2. TEM image of modified ferrous-ferric-Silicon dioxide- $\mathrm{NH}_{2}$

Batch procedure

To study the ability of the adsorbents (ferrous-ferric-silicon dioxide-NH2) to remove lead ions from water, all samples were tested under the different contact times $(1,1.5,2,2.5,3 \mathrm{~h})$ at $\mathrm{pH}=6$. Briefly, $2 \mathrm{~g}$ ferrous-ferric-silicon dioxide- $\mathrm{NH}_{2}$ nanocomposites soaked in $50 \mathrm{ml}$ tubes contain $30 \mathrm{ppb}$ lead ions solution. Then tubes were shaken at $150 \mathrm{rpm}$ at room temperature in different contact times. Treated solutions were filtered for atomic absorption analysis. Figures 3 shows the lead ions removal capacity at different contact times by applying atomic absorption analysis. As shown, by increasing the contact time, the number of lead ions that attached to ferrous-ferric-silicon dioxide- $\mathrm{NH}_{2}$ NPs under sorption mechanisms increases. Subsequently, the adsorption reached equilibrium after $2 \mathrm{~h}$ (78wt\%), and further increase had no effect on the adsorption capacity attributable to the reduction of the number of available active sites on surface of nanoparticles and lead ions concentration. Moreover, the capacity of modified absorbent to react with lead ions through adsorption and an ion exchange mechanism demonstrated higher removal of lead from water. Additionally, the prepared adsorbent could be separated from an aqueous solution by using an external magnet field (Figure 4).

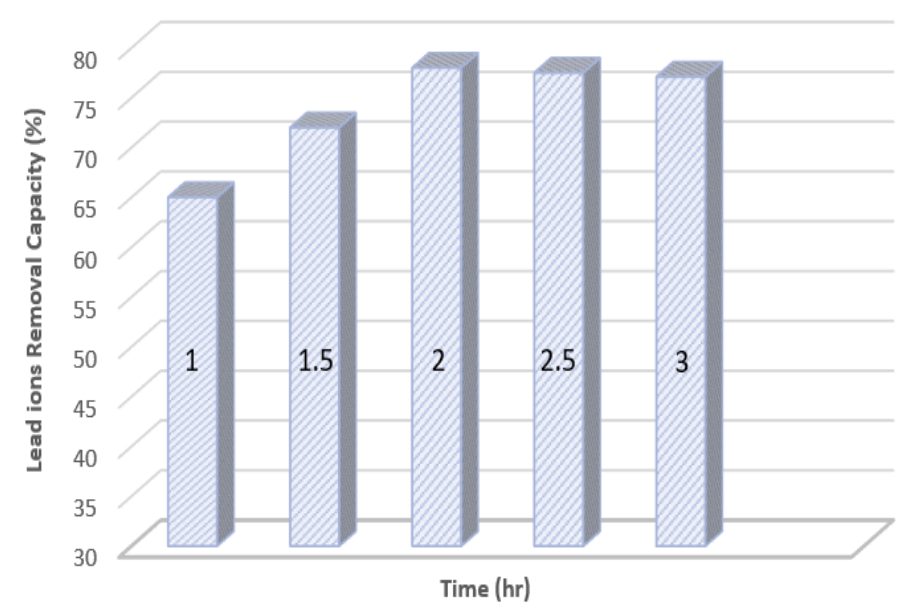

Figure 3. lead ions removal capacity at different contact times 

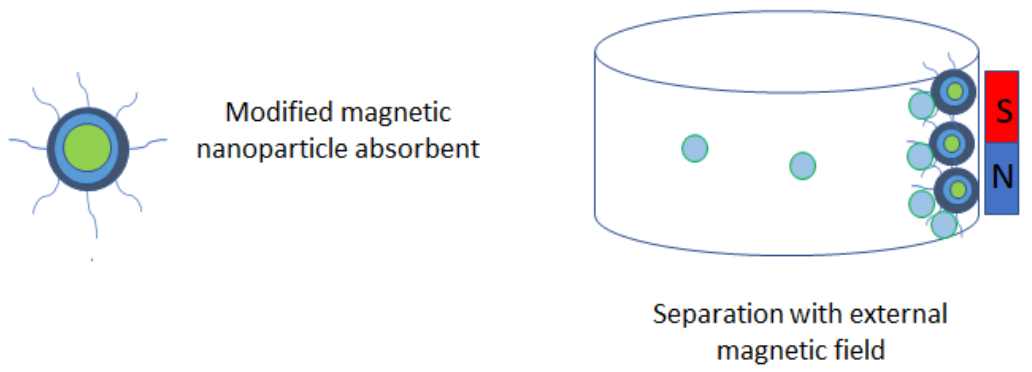

Figure 4. Separation of absorbent from aqueous solution by using external magnetic field

\section{Conclusions}

Ferrous-ferric-silicon dioxide- $\mathrm{NH}_{2}$ nanoparticles were synthesized by sol-gel method by the reaction of ferric and ferrous in ammonia solution to investigate the removal of lead ions from aqueous solution. Surface morphology of synthesized nanoparticles were characterized by FT-IR and TEM analysis and the results showed that the synthesized nanoparticles have nanosized (below $100 \mathrm{~nm}$ ) and core-shell structure. When the contact time increased, the number of lead ions that attached to the surface of ferrous-ferric-silicon dioxide- $\mathrm{NH}_{2} \mathrm{NPs}$ under sorption mechanisms increased. Modified ferrous-ferricsilicon dioxide- $\mathrm{NH}_{2}$ nanoparticles showed adsorption performance with a removal efficiency of $78 \mathrm{wt} \%$ during a contact time of $2 \mathrm{~h}$ at $\mathrm{pH}=6$ (at room temperature). In other words, the ability of modified ferrous-ferric oxide-silicon dioxide- $\mathrm{NH}_{2}$ to react with lead ions through adsorption and an ion exchange mechanism illustrated higher removal capacities of lead from an aqueous solution. Additionally, prepared adsorbent could be able to separate from an aqueous solution by using an external magnet field.

Conflicts of Interest: The author declares no conflict of interest.

\section{References}

1. Meng L., et al., Lead removal from water by a newly isolated Geotrichum candidum LG-8 from Tibet kefir milk and its mechanism. Chem 2020 259,127507.

2. Aidil M.E., et al.; Modified oil palm industry solid waste as a potential adsorbent for lead removal, Environ Chem Eco 2021 3, 1-7.

3. Huy, D.H., et al., Removal mechanisms of cadmium and lead ions in contaminated water by stainless steel slag obtained from scrap metal recycling, J Water Process Eng, 2020 36,101369.

4. Moghaddam, S.T., et al., High Removal Capacity of Arsenic from Drinking Water Using Modified Magnetic Polyurethane Foam Nanocomposites. J Polym Environ 2019 27(7), 1497-1504. https://doi.org/10.1007/s10924-019-01446-7.

5. Moghaddam, S.T.; et al., Effect of Novel Magnetic Nanoparticles on Morphology properties of Polyurethane Foam. The 21st International Electronic Conference on Synthetic Organic Chemistry Sciforum Electronic Conference Series 2017, 21. https://doi.org/10.3390/ecsoc-21-04828.

6. Moghaddam, S.T.; et al., Reinforced magnetic polyurethane rigid (PUR) foam nanocomposites and investigation of thermal, mechanical, and sound absorption properties. J Thermplast Comp Mater 2018, https://doi.org/10.1177/0892705718798008.

7. Kayani, Z.N., et al., Synthesis of Iron Oxide Nanoparticles by Sol-Gel Technique and Their Characterization. IEEE Transactions on Magnetics 2014 50, 1-4.

8. Sahoo J.k., et al.; EDTA functionalized magnetic nanoparticle as a multifunctional adsorbent for Congo red dye from contaminated water. AIP Conference Proceedings 2017 1832, 050087 https://doi.org/10.1063/1.4980320.

9. Kurtan, U., et al., Fabrication and characterization of $\mathrm{Fe}_{3} \mathrm{O}_{4} @$ APTES@PAMAM-Ag highly active and recyclable magnetic nanocatalyst: Catalytic reduction of 4-nitrophenol, Mater Res Bull, 2014 60, 79-87. 